\title{
Homeostatic Synaptic Plasticity through Changes in Presynaptic Calcium Influx
}

\author{
CongJian Zhao, ${ }^{\star}$ Elena Dreosti, ${ }^{\star}$ and Leon Lagnado \\ Medical Research Council Laboratory of Molecular Biology, Cambridge CB2 0QH, United Kingdom
}

Chronic perturbations of electrical activity within neural circuits lead to compensatory changes in synaptic strength collectively termed homeostatic synaptic plasticity. The postsynaptic mechanisms underlying these modifications have been characterized in some detail, but the presynaptic mechanisms that alter the efficiency of evoked neurotransmitter release are less clear. To investigate the role of presynaptic calcium influx, we have combined the use of two fluorescent proteins in cultured hippocampal neurons: a calcium reporter localized to synaptic vesicles, SyGCaMP2, and a reporter of vesicle fusion, SypHy. We find that a decrease in the activity of the network causes an increase in the amount of calcium entering the synaptic bouton in response to an action potential and an increase in the probability of vesicle fusion. Homeostatic changes in release probability varied as the third power of calcium influx. These results indicate that changes in the number and/or function of presynaptic calcium channels are major determinants of homeostatic changes in synaptic strength.

\section{Introduction}

The operation of neural circuits involves continuous variations in the electrical excitability of neurons and the strength of the synaptic connections between them, but this activity must be maintained within certain bounds if the circuit is to operate stably. Regulation occurs through negative feedback mechanisms that control synaptic strength and membrane excitability in the face of long-term perturbations (Turrigiano et al., 1998; Murthy et al., 2001; Burrone et al., 2002; Frank et al., 2006). Changes in synaptic function that occur in this context are termed homeostatic synaptic plasticity (HSP) or synaptic scaling (Turrigiano, 2008).

Homeostatic synaptic plasticity has been studied extensively in central mammalian neurons in culture (Turrigiano et al., 1998; Burrone and Murthy, 2003; Wierenga et al., 2006) and in vivo (Maffei et al., 2004, 2006; Goel and Lee, 2007), as well as in the neuromuscular junction (NMJ) of flies (Davis, 2006). A consistent response to manipulation of global network activity is a change in quantal amplitude, primarily reflecting changes in the number of neurotransmitter receptors postsynaptically (for review, see Turrigiano, 2008). A second component of HSP is thought to be presynaptic - regulation of the probability of vesicle release (Pr) in response to an action potential (AP) (Murthy et al., 2001; Thiagarajan et al., 2005). The most convincing evidence for homeostatic regulation of evoked release comes from studies of the fly NMJ, where genetics reveal a dependence on $\mathrm{Ca}_{\mathrm{v}} 2.1$

\footnotetext{
Received Dec. 20, 2010; revised March 8, 2011; accepted April 3, 2011.

Author contributions: E.D. and L.L. designed research; C.Z., E.D., and L.L. performed research; C.Z., E.D., and L.L. analyzed data; C.Z., E.D., and L.L. wrote the paper.

This work was supported by the Medical Research Council and the Wellcome Trust.

${ }^{*}$ C.Z. and E.D. contributed equally to this work.

The authors declare no competing financial interests.

Correspondence should be addressed to Leon Lagnado, MRC Laboratory of Molecular Biology, Hills Road, Cambridge CB2 0QH, UK. E-mail: II1@mrc-Imb.cam.ac.uk.

DOI:10.1523/JNEUROSCI.6636-10.2011

Copyright $\odot 2011$ the authors $\quad 0270-6474 / 11 / 317492-05 \$ 15.00 / 0$
}

(P/Q) $\mathrm{Ca}^{2+}$ channels (Frank et al., 2006, 2009). It is much more difficult to use electrophysiology to measure release probability at individual synaptic connections in the CNS of mammals. The best attempts to detect homeostatic changes in Pr have applied an optical approach to cultured neurons in which FM dyes are loaded into synaptic vesicles and rates of destaining are measured during prolonged stimulation. In cultures of neocortical or hippocampal neurons, inhibition of spiking activity by TTX is followed by an increase in the probability of release, as long as TTX is applied after neurons have spent at least $14 \mathrm{~d}$ in vitro (Murthy et al., 2001; Wierenga et al., 2006; Branco et al., 2008).

How might homeostatic changes in release probability occur? Two basic possibilities are changes in the presynaptic calcium signal triggered by an action potential, or changes in the efficiency of vesicle fusion in response to calcium. It is very difficult to assess electrical events in small presynaptic terminals by electrophysiological recordings, so we used SyGCaMP2, a genetically encoded calcium indicator localized to synaptic vesicles (Dreosti et al., 2009; Dreosti and Lagnado, 2011). This new optical approach allows calcium influx in response to a single action potential to be quantitated at individual synaptic boutons. Using cultured hippocampal neurons, we find that a chronic decrease in spiking activity caused by treatment with TTX is accompanied by an increase in the mean amplitude of the unitary calcium transient generated by an action potential, while a chronic increase in activity caused by treatment with gabazine is accompanied by a smaller calcium transient. In parallel, we measured changes in release probability using SypHy, a fluorescent protein that reports synaptic vesicle fusion with much improved signal-to-noise ratio compared with FM dyes (Granseth et al., 2006). We find a third-power relation between calcium influx and release probability in cultures displaying different levels of activity. These results demonstrate that small changes in the number and/or function of presynaptic calcium channels have large relative ef- 
fects on synaptic strength. We propose that presynaptic calcium channels are a key control point for the presynaptic component of homeostatic plasticity in mammalian neurons.

\section{Materials and Methods}

Primary neuronal culture and transfection. Dissociated hippocampal neurons were prepared from embryonic day 19 mice in accordance with Home Office regulations (Granseth et al., 2006). Cultures were allowed to grow on glass coverslips for $14-17 \mathrm{~d}$ before experiments were conducted. Neurons were transfected to express SyGCaMP2 (Dreosti et al., 2009) or SypHy (Granseth et al., 2006) using Lipofectamine 2000 (Invitrogen) in minimal essential medium applied after $7 \mathrm{~d}$ in vitro. At least $7 \mathrm{~d}$ were allowed between transfection and imaging so as to obtain a high and localized expression of the reporters at synapses. To induce chronic perturbation of synaptic activity, cultures were treated with TTX $(0.5 \mu \mathrm{M})$ or gabazine $(10 \mu \mathrm{M})$ for $24-48 \mathrm{~h}$ before imaging. Experiments using TTX, gabazine, and controls were performed in parallel on sister cultures from the same preparation.

Imaging synaptic reporters. All experiments were conducted as described previously (Granseth et al., 2006). Coverslips were mounted on a customized imaging chamber (RC-25F, Warner Instruments) comprising of two parallel platinum wires $3 \mathrm{~mm}$ apart to evoke APs through electric field stimulation (20 mA, $1 \mathrm{~ms}$ pulses). During experiments, neurons were perfused with a buffer, $\mathrm{pH} 7.4$, containing the following (in mM): $136 \mathrm{NaCl}, 2.5 \mathrm{KCl}, 10 \mathrm{HEPES}, 1.3 \mathrm{MgCl}_{2}, 10$ Glucose, $2 \mathrm{CaCl}_{2}$, all from Sigma-Aldrich. To reduce spontaneous synaptic transmission, 10 $\mu \mathrm{M}$ CNQX and $50 \mu \mathrm{M}$ DL-APV (Tocris Cookson) were also present in the medium. Before every experiment, neurons treated with TTX or gabazine were washed for 15-20 min. Images were taken using a CCD camera (Cascade 512B, Photometrics) mounted on an inverted microscope (Diaphot 200, Nikon) with a $40 \times, 1.3$ numerical aperture oil-immersion objective. The filter set comprised a 475AF40 excitation filter, a 505DRLP dichroic mirror, and a 535AF45 emission filter (Omega). Illumination was obtained using a light-emitting diode (Luxeon K2, blue) driven from a constant current source at $1500 \mathrm{~mA}$, and the beam was controlled by a shutter (Uniblitz VMM-D3, Vincent Associates). For all experiments, images were captured in Frame Transfer mode at a depth of 16 bits to a Macintosh G4 computer using IPLab software (BD Biosciences). Image sequences of $128 \times 128$ pixels (binned $2 \times 2$ ) were acquired at $20 \mathrm{~Hz}$ using $45 \mathrm{~ms}$ integration times for SyGCaMP2 and at $5 \mathrm{~Hz}$ using $195 \mathrm{~ms}$ exposure for SypHy.

Electric field stimulation of cultured neurons. The stimulus we used to trigger a single $\mathrm{AP}$ was a $1 \mathrm{~ms}$ application of a $10 \mathrm{~V}$ potential difference between platinum wires $\sim 3-4 \mathrm{~mm}$ apart, applied using a Grass stimulator. To monitor synaptic calcium responses using SyGCaMP2, a variable number of pulses were delivered at $20 \mathrm{~Hz}$. To measure Pr using SypHy, we delivered 10 single pulses at $45 \mathrm{~s}$ intervals, followed by a single train of 40 pulses at $20 \mathrm{~Hz}$.

All the imaging experiments reported here were performed with excitatory transmission inhibited by the glutamate receptor (GluR) blockers CNQX $(10 \mu \mathrm{M})$ and APV $(50 \mu \mathrm{M})$. It is generally thought that under these conditions a $1 \mathrm{~ms}$ field stimulus will only trigger one AP (Gandhi and Stevens, 2003). We checked whether this was the case under our experimental conditions by making current-clamp recordings using an Axopatch 200A amplifier (Molecular Devices) and an intracellular solution containing the following (in $\mathrm{mm}$ ): potassium gluconate $135, \mathrm{NaCl} 7$, $\mathrm{MgCl}_{2} 2, \mathrm{Na}_{2} \mathrm{ATP} 2$, NaGTP 0.3, HEPES 10, pH 7.3. In the absence of GluR blockers, polysynaptic activity was sometimes strong enough to trigger "secondary spikes" within a few tens of milliseconds of the first spike triggered directly by the $1 \mathrm{~ms}$ field stimulus (Fig. 1). Secondary spikes were observed in one of three cultures made hypoexcitable by pretreatment with gabazine, zero of two control cultures, and three of four cultures made hyperexcitable by pretreatment with TTX. In all four cases in which secondary spikes were observed in the absence of GluR blockers, they were immediately abolished by addition of $10 \mu \mathrm{M}$ CNQX and $50 \mu \mathrm{M}$ DL-APV. An example of this behavior in a culture pretreated with TTX is shown in Figure 1. In all nine cultures in which recordings were made in the presence of GluR blockers, a single stimulus reliably triggered one, and only one, spike.
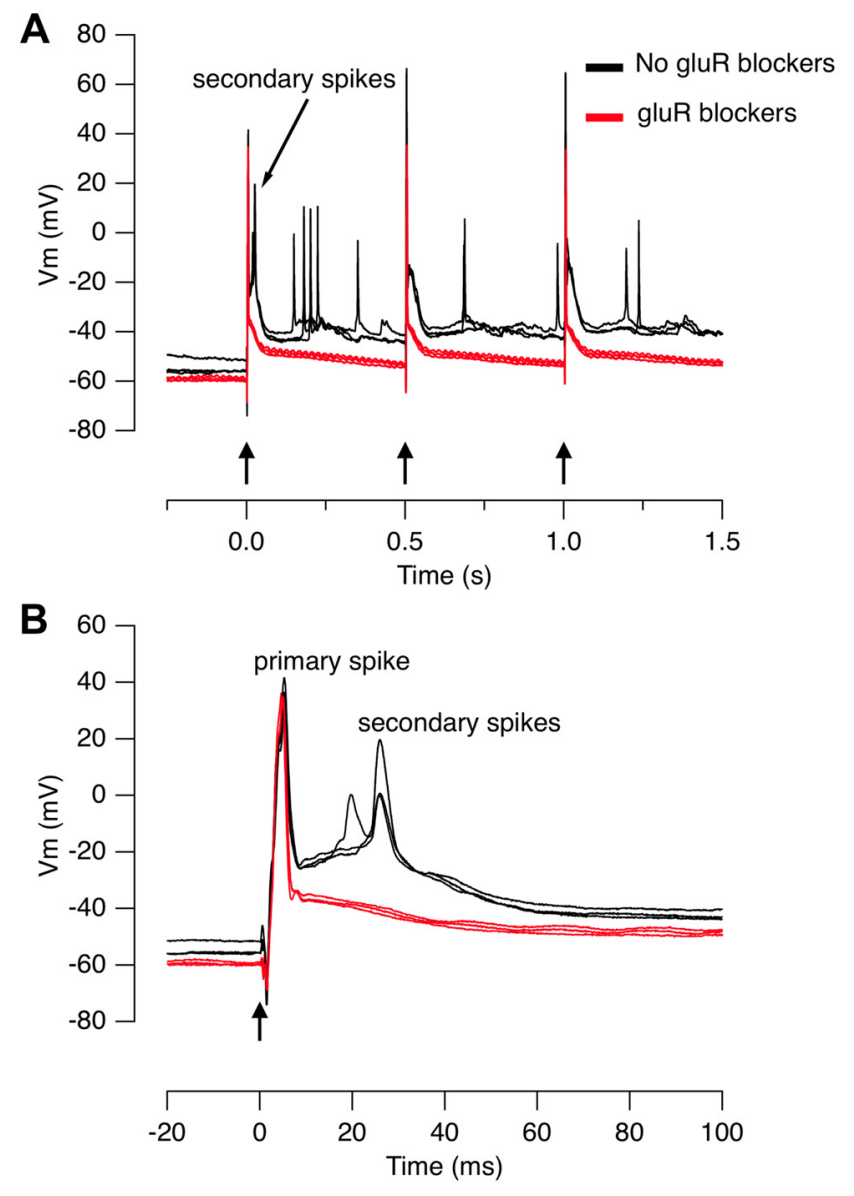

Figure 1. Glutamate receptor blockers prevented spikes triggered by polysynaptic activity. $A$, Current-clamp recording from a neuron in a culture pretreated with TTX for $2 \mathrm{~d}$ and then washed in normal medium for $20 \mathrm{~min}$. Each trace shows the response to a sequence of three field stimuli (1 ms) at 0.5 s intervals: three trials in the absence of blockers (black) are superimposed on three trials obtained within 2 min of adding $10 \mu \mathrm{M}$ CNQX and $50 \mu \mathrm{M} \mathrm{DL}-\mathrm{APV}$ (red). Holding current was $0 \mathrm{pA}$. For each trace, the stimulus artifact was subtracted by delivering a pulse with the direction of the electric field reversed, adding the two records, and dividing by two. Secondary spikes triggered by polysynaptic activity (arrowed) only occurred after the first stimulus of the sequence. Addition of GluR blockers suppressed both spontaneous synaptic activity and secondary spikes. $\boldsymbol{B}$, The responses to the first stimulus in $\boldsymbol{A}$ on an expanded time scale. In the presence of GluR blockers, a single stimulus reliably triggered one, and only one, spike.

Image analysis. All image sequences were analyzed using customwritten macros in Igor Pro (WaveMetrics) as previously described (Granseth et al., 2006). Briefly, responding synapses were identified on "difference images" highlighting the stimulus-dependent increase of fluorescence. Synaptic responses were obtained by placing square regions of interest (ROIs) on synapses and measuring the average fluorescence per each ROI over time. ROIs were $2.3 \times 2.3 \mu \mathrm{M}$ for SyGCaMP2 measurements and $4.6 \times 4.6 \mu \mathrm{M}$ for SypHy measurements. Only synapses with fluorescent responses greater than SNR 2 in response to 10 APs (for SyGCaMP2) or 40 APs (for SypHy) were considered. To subtract the local background signal, the intensity of an ROI displaced in the $x$ - or $y$-direction by $2.3 \mu \mathrm{m}$ was also measured. Signals were quantified as $\Delta F / F_{0}$, where $F_{0}$ was the baseline fluorescence.

\section{Results}

Homeostatic changes in presynaptic calcium influx

To investigate the calcium transient at individual active zones, we used cultured hippocampal neurons expressing SyGCaMP2 (Dreosti et al., 2009), a genetically encoded calcium reporter specifically localized to synaptic vesicles (Fig. $2 A$ ). Figure $2 A$ shows 
A
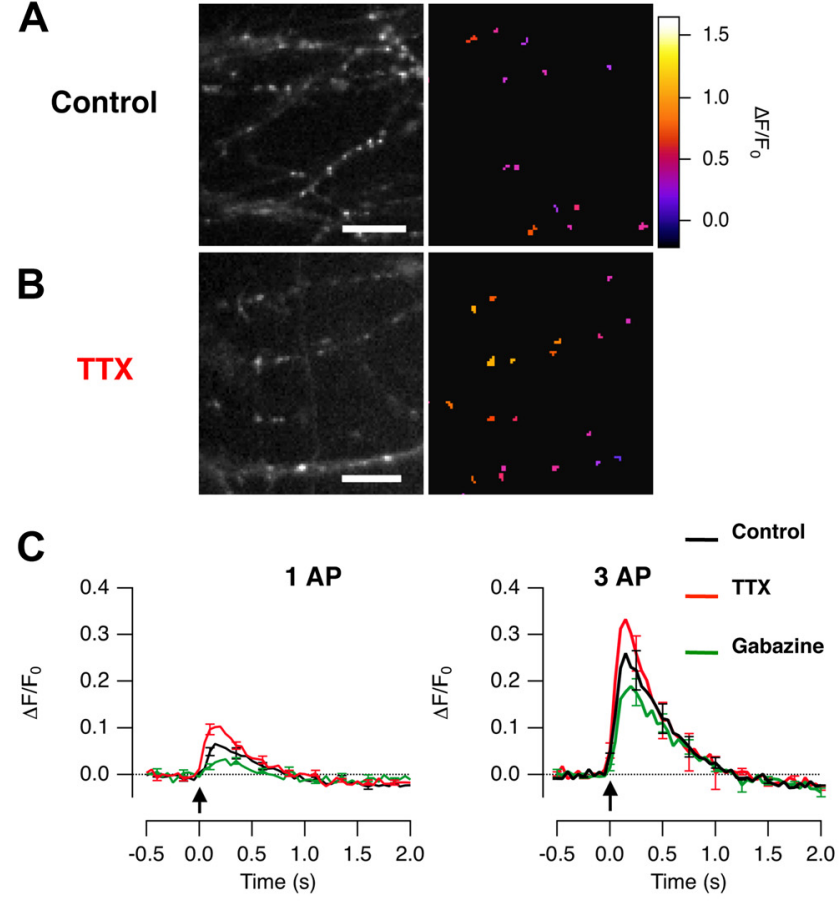

D

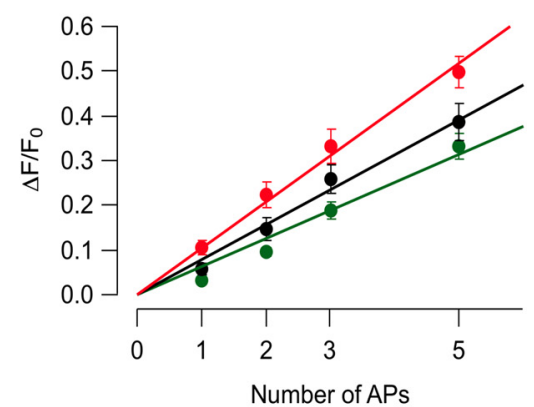

Figure 2. Homeostatic changes in presynaptic calcium influx detected with SyGCaMP2. A, Fluorescence image of cultured hippocampal neurons expressing SyGCaMP2 (left) and pseudocolored images showing the relative change in fluorescence $0.3 \mathrm{~s}$ from the beginning of a train of 3 APs delivered at $20 \mathrm{~Hz}$. B. The response to 3 APs was larger in a culture treated with TTX for $2 \mathrm{~d}$. Note the variability in the amplitude of the presynaptic calcium signal between different boutons. Scale bar, $10 \mu \mathrm{m}$. C, Averaged SyGCaMP2 response to $1 \mathrm{AP}$ (left) and 3 APs (right). Control cultures are black ( $n=805$ synapses), TTX-treated cultures are red $(n=1200)$, and gabazine-treated cultures are green $(n=386)$. Error bars are SEM. $\boldsymbol{D}$, Peak amplitude of the SyGCaMP2 signal plotted as a function of the number of APs delivered at $20 \mathrm{~Hz}$. Line fits have the following slopes: control (black), $0.078 \pm 0.003 ;$ TTX (red), $0.104 \pm 0.003 ;$ gabazine (green), $0.063 \pm 0.004$. Error bars are SEM.

the response of SyGCaMP2 to 3 APs delivered at $20 \mathrm{~Hz}$ in a culture under control conditions, and Figure $2 B$ shows a response to the same stimulus in a culture pretreated with 500 nM TTX for $2 \mathrm{~d}$ to block spiking activity. The amplitude of the SyGCaMP2 signal reflects the amount of calcium entering the bouton (Dreosti et al., 2009). Although varying between different boutons (Fig. $2 \mathrm{~A}$ ), presynaptic calcium influx was significantly larger in the TTX-treated network when measured in response to 1 or $3 \mathrm{AP}$ (Fig. 2C). To investigate the effect of an increase in network activity, cultures were pretreated for 1-2 d with $10 \mu \mathrm{M}$ gabazine, which acts by blocking $\mathrm{GABA}_{\mathrm{A}}$ receptors mediating inhibitory transmission. Gabazine had the opposite effect to TTX, causing a decrease in presynaptic calcium influx (Fig. 2C).

We have previously demonstrated that the relation between the peak amplitude of the SyGCaMP2 signal and the number of
APs delivered at $20 \mathrm{~Hz}$ is linear up to a total of $\sim 8$ APs in untreated cultures (Dreosti et al., 2009). The same was found when network activity was chronically increased or decreased (Fig. 2D). The slope of this relation therefore provided a more reliable method of comparing the average amplitude of the unitary calcium signal elicited by a single AP. The peak SyGCaMP2 signal per $\operatorname{AP}\left(\Delta F / F_{0}\right)$ was $0.078 \pm 0.003$ under control conditions, $0.104 \pm 0.003$ immediately after TTX treatment and $0.063 \pm$ 0.004 after gabazine treatment.

\section{Homeostatic changes in release probability}

How do these changes in calcium influx impact on release probability? To monitor synaptic vesicle fusion across a large population of synapses, we imaged SypHy, which provides greater sensitivity than FM dyes (Granseth et al., 2006). The images in Figure $3 A$ compare the response of SypHy in cultures treated with TTX and gabazine, first to $40 \mathrm{AP}$ at $20 \mathrm{~Hz}$ (middle), then to $1 \mathrm{AP}$ (right, averaged over 10 trials). The average number of vesicles released by $1 \mathrm{AP}$ varied widely between different boutons, but was on average threefold smaller in the culture pretreated with gabazine (Fig. 3B, right). The suppression of evoked release in more active networks could not be explained simply by a decrease in the total number of vesicles within each bouton, because the average response to a train of $40 \mathrm{APs}$ at $20 \mathrm{~Hz}$ was only slightly reduced under the two conditions (Fig. 3B, left). Thus, HSP involved changes in the probability that a single spike would trigger fusion of a vesicle, but with little effect on the proportion of vesicles within the terminal that could be released by a longer train.

A stimulus of $40 \mathrm{APs}$ at $20 \mathrm{~Hz}$ is thought to completely deplete the readily releasable pool (RRP) of vesicles in hippocampal synapses (Rosenmund and Stevens, 1996). The average release probability of an individual vesicle within the RRP in response to a single spike, Pr, was therefore defined as the ratio of the peak responses to 1 and $40 \mathrm{APs}$ (Granseth et al., 2006). Figure 3C shows the distribution of $\mathrm{Pr}$ in control, gabazine-treated, and TTX-treated cultures. Values of Pr were widely dispersed, reflecting the known heterogeneity in synaptic strength observed in cultures and slices (Murthy et al., 1997). The variability in $\mathrm{Pr}$ could be described as a gamma distribution

$$
f(x)=A \frac{(x / \beta)^{\alpha-1} \exp (-x / \beta)}{\beta \Gamma(\alpha)},
$$

with shape parameter $\alpha=4$. The larger the scale parameter, $\beta$, the more spread out the distribution. The values of $\beta$ in gabazine, control, and TTX were $0.0077 \pm 0.0005,0.0147 \pm 0.0006$, and $0.0206 \pm 0.0006$, respectively.

The presynaptic mechanisms underlying these variations in $\mathrm{Pr}$ are not well understood, but variations in calcium influx are a strong candidate. We therefore investigated how HSP affected heterogeneities in the presysnaptic calcium signal. Figure $3 D$ shows the distributions of the peak SyGCaMP2 response elicited by 1 AP under our three experimental conditions. The variability in presynaptic calcium influx could be described as a gamma distribution with shape parameter $\alpha=2$. The values of $\beta$ in gabazine, control, and TTX were $0.103 \pm 0.013,0.127 \pm 0.004$, and $0.17 \pm 0.004$, respectively. Thus, the less active the network, the greater the heterogeneity in $\operatorname{Pr}$ (Fig. $3 C$ ) and the presynaptic calcium signal (Fig. 3D). 
A

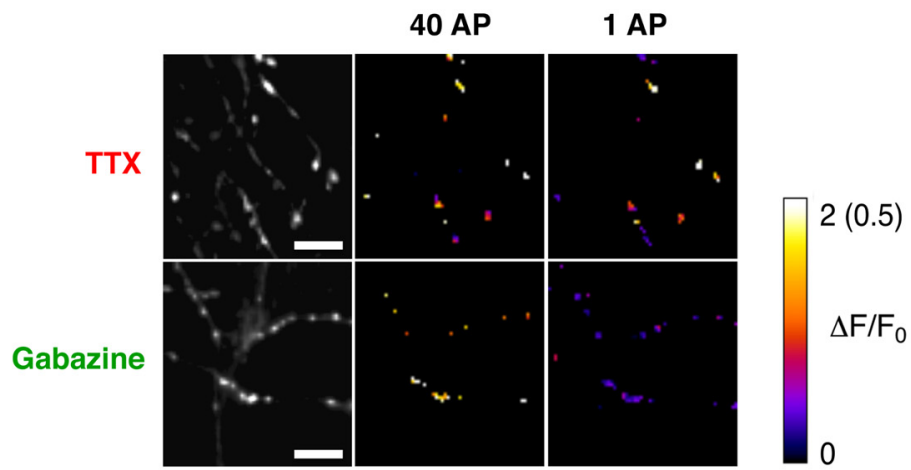

B
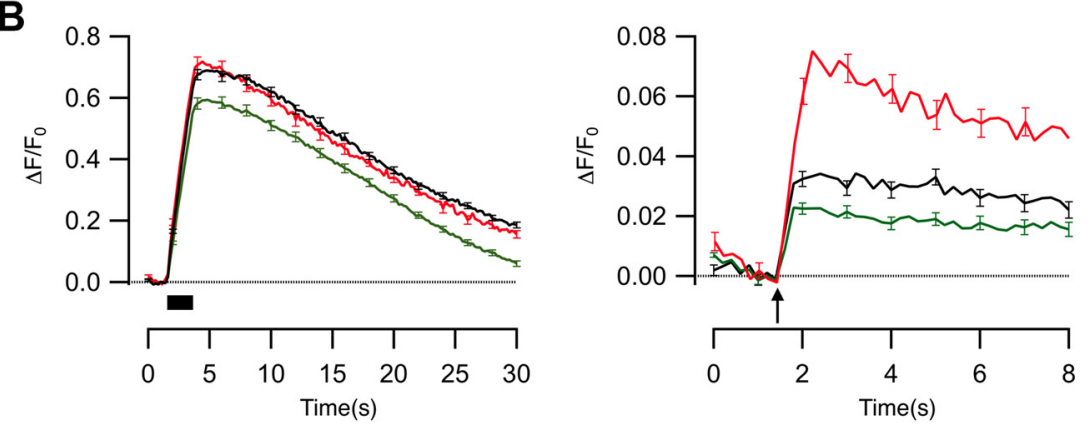

C

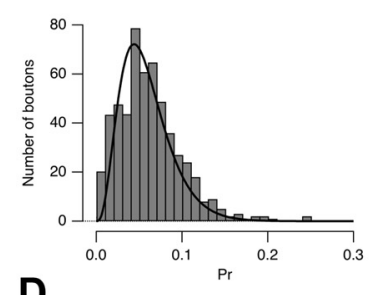

D

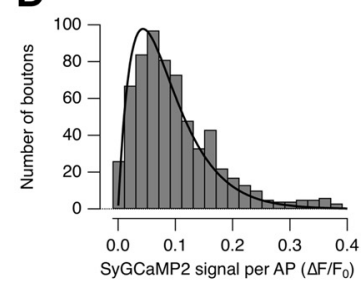

Gabazine
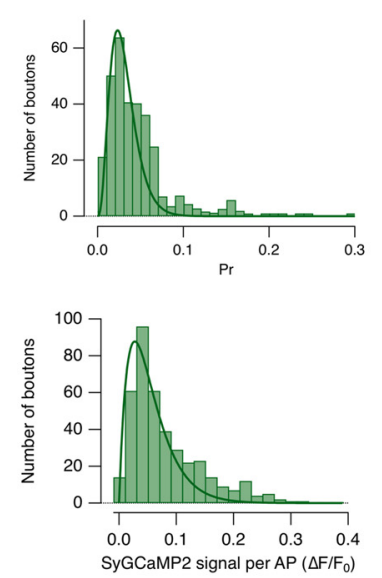

TTX
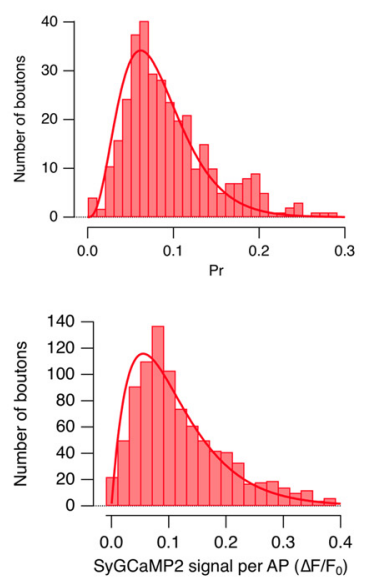

Figure 3. Homeostatic changes in evoked vesicle fusion detected with SypHy. A, Fluorescence image of cultured hippocampal neurons expressing SypHy (left) and pseudocolored images showing the relative change in fluorescence in response to $40 \mathrm{APs}$ at 20 $\mathrm{Hz}$ (middle) and $1 \mathrm{AP}$ (right). Top row: TTX-treated culture. Bottom row: gabazine-treated culture. Note that the pseudocolor scale has a maximum (white) of 2 for images showing the response to $40 \mathrm{APs}$, but only 0.5 for the images showing the response to $1 \mathrm{AP}$. $B$, Relative change in SypHy fluorescence in response to a train of $40 \mathrm{APs}$ at $20 \mathrm{~Hz}$ (left) and just $1 \mathrm{AP}$ (right). Note the different scales. Black: control ( $n=945$ synapses). Red: TTX-treated cultures ( $n=470$ synapses). Green: gabazine-treated $(n=793)$. Bars are SEM. C, The distribution of release probabilities (Pr) in control, gabazine-treated, and TTX-treated cultures. Pr was calculated as the ratio of the peak responses to 1 and 40 APs for each individual synapse. Data were described using a gamma distribution, all with a shape parameter of $\alpha=4$. Values of the scale parameter $\beta$ were as follows: control, $0.0147 \pm 0.0006$; gabazine, $0.0077 \pm$ $0.0005 ; \mathrm{TTX}, 0.0206 \pm 0.0006$. D, The distribution of SyGCaMP2 signal per AP. Data were described using a gamma distribution, all with $\alpha=2$. The scale parameter $\beta$ was as follows: control, $0.127 \pm 0.004$ ( $n=667$ synapses); TTX, $0.17 \pm 0.004$ ( $n=938$ ); gabazine, $0.103 \pm 0.013(n=404)$.

\section{A third-power relation between homeostatic changes in presynaptic calcium influx and release probability}

The relation between the average values of $\operatorname{Pr}$ within active synapses and the SyGCaMP2 signal per AP is shown in Figure 4, with each point being calculated within one of the three experimental conditions. Pr was proportional to the amount of presynaptic calcium influx raised to the power of 3, consistent with a highly cooperative relation between calcium and release rate (Katz and Miledi, 1970). This relation highlights an important point: relatively small changes in calcium influx caused large relative changes in the rate of vesicle release.

\section{Discussion}

In this study, we have directly measured changes in presynaptic calcium influx in response to long-term changes in network activity. The single synapse/single AP resolution of SyGCaMP2 and improved sensitivity of SypHy compared with FM dyes lead to the following mechanistic insights: (1) the wide heterogeneity in Pr reflects heterogeneity in calcium influx between different boutons; (2) homeostatic changes in presynaptic function involve shifts in the distributions of $\mathrm{Pr}$ and the amount of calcium influx per spike; and (3) there is a third-power law describing the relation between Pr and presynaptic calcium influx when measurements are averaged over populations of boutons under different levels of network activity. These results lead to the conclusion that the presynaptic calcium signal is a key control point for regulating synaptic strength in response to chronic changes in network activity.

A cooperative (fourth-power relation) between calcium influx and release probability has been noted at a number of synapses, including hippocampal boutons (Katz and Miledi, 1970; Sinha et al., 1997). The fact that a similar relation is observed when calcium influx is altered in response to chronic changes in activity (Fig. 4) suggests that the presynaptic component of homeostatic plasticity is primarily determined by a change in the amount of calcium entering in response to a spike rather than changes in the molecular machinery determining the efficiency with which calcium triggers vesicle fusion. The cooperative relation between calcium influx and vesicle fusion will allow small changes in the number of presynaptic calcium channels to have large relative effects on synaptic strength.

The mechanisms by which presynaptic calcium influx is altered during homeostatic synaptic plasticity remain to be determined. Possibilities include the fol-

lowing: changes in the number of voltagesensitive calcium channels in the presynaptic membrane controlled by trafficking (Jarvis and Zamponi, 2007); regulation of calcium channel function, for instance through neuronal calcium-sensor proteins, which also mediate short-term plasticity (Mochida et al., 2008); and regulation of presynaptic sodium and potassium currents contributing to the voltage trajectory in the presynaptic compartment. The last idea is very difficult to test 


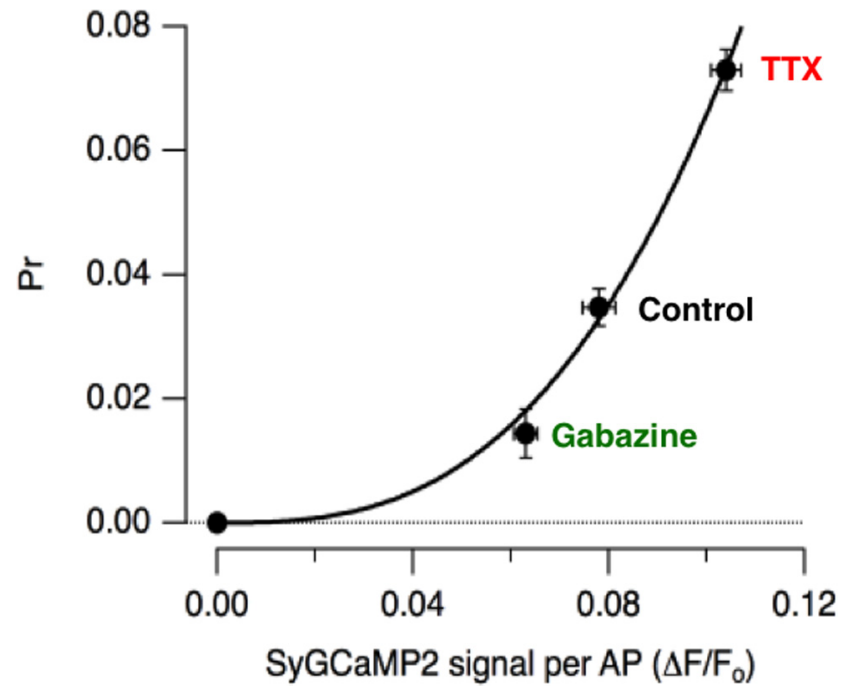

Figure 4. Homeostatic changes in evoked vesicle fusion reflect a cooperative action of calcium. The relation between Pr measured with SypHy and presynaptic calcium influx measured with SyGCaMP2 is shown. The curve fitted through the points is a power function of the form Axy, with $y=2.8 \pm 0.3$. Error bars are SEM.

using electrophysiology because the presynaptic compartments of most mammalian neurons are very small: the best recordings of presynaptic sodium and potassium have been made at the Calyx of Held, a giant synapse that can be patched and voltageclamped (Dodson and Forsythe, 2004; Leao et al., 2005). It is because of the difficulties involved in assessing electrical events in small presynaptic terminals that we developed the use of SyGCaMP2 to monitor presynaptic calcium influx optically. This study demonstrates how genetically encoded reporters such as SyGCaMP2 and SypHy can be used to study mechanisms of synaptic plasticity (Dreosti and Lagnado, 2011).

\section{References}

Branco T, Staras K, Darcy KJ, Goda Y (2008) Local dendritic activity sets release probability at hippocampal synapses. Neuron 59:475-485.

Burrone J, Murthy VN (2003) Synaptic gain control and homeostasis. Curr Opin Neurobiol 13:560-567.

Burrone J, O’Byrne M, Murthy VN (2002) Multiple forms of synaptic plasticity triggered by selective suppression of activity in individual neurons. Nature 420:414-418.

Davis GW (2006) Homeostatic control of neural activity: from phenomenology to molecular design. Annu Rev Neurosci 29:307-323.

Dodson PD, Forsythe ID (2004) Presynaptic $\mathrm{K}^{+}$channels: electrifying regulators of synaptic terminal excitability. Trends Neurosci 27:210-217.

Dreosti E, Lagnado L (2011) Optical reporters of synaptic activity in neural circuits. Exp Physiol 96:4-12.
Dreosti E, Odermatt B, Dorostkar MM, Lagnado L (2009) A genetically encoded reporter of synaptic activity in vivo. Nat Methods 6:883-889.

Frank CA, Kennedy MJ, Goold CP, Marek KW, Davis GW (2006) Mechanisms underlying the rapid induction and sustained expression of synaptic homeostasis. Neuron 52:663-677.

Frank CA, Pielage J, Davis GW (2009) A presynaptic homeostatic signaling system composed of the Eph receptor, ephexin, Cdc42, and CaV2.1 calcium channels. Neuron 61:556-569.

Gandhi SP, Stevens CF (2003) Three modes of synaptic vesicular recycling revealed by single-vesicle imaging. Nature 423:607-613.

Goel A, Lee HK (2007) Persistence of experience-induced homeostatic synaptic plasticity through adulthood in superficial layers of mouse visual cortex. J Neurosci 27:6692-6700.

Granseth B, Odermatt B, Royle SJ, Lagnado L (2006) Clathrin-mediated endocytosis is the dominant mechanism of vesicle retrieval at hippocampal synapses. Neuron 51:773-786.

Jarvis SE, Zamponi GW (2007) Trafficking and regulation of neuronal voltage-gated calcium channels. Curr Opin Cell Biol 19:474-482.

Katz B, Miledi R (1970) Further study of the role of calcium in synaptic transmission. J Physiol 207:789-801.

Leão RM, Kushmerick C, Pinaud R, Renden R, Li GL, Taschenberger H, Spirou G, Levinson SR, von Gersdorff H (2005) Presynaptic $\mathrm{Na}^{+}$Channels: locus, development, and recovery from inactivation at a high-fidelity synapse. J Neurosci 25:3724-3738.

Maffei A, Nelson SB, Turrigiano GG (2004) Selective reconfiguration of layer 4 visual cortical circuitry by visual deprivation. Nat Neurosci 7 : 1353-1359.

Maffei A, Nataraj K, Nelson SB, Turrigiano GG (2006) Potentiation of cortical inhibition by visual deprivation. Nature 443:81-84.

Mochida S, Few AP, Scheuer T, Catterall WA (2008) Regulation of presynaptic $\mathrm{Ca}(\mathrm{V}) 2.1$ channels by $\mathrm{Ca} 2+$ sensor proteins mediates short-term synaptic plasticity. Neuron 57:210-216.

Murthy VN, Sejnowski TJ, Stevens CF (1997) Heterogeneous release properties of visualized individual hippocampal synapses. Neuron 18:599-612.

Murthy VN, Schikorski T, Stevens CF, Zhu Y (2001) Inactivity produces increases in neurotransmitter release and synapse size. Neuron 32: 673-682.

Rosenmund C, Stevens CF (1996) Definition of the readily releasable pool of vesicles at hippocampal synapses. Neuron 16:1197-1207.

Sinha SR, Wu LG, Saggau P (1997) Presynaptic calcium dynamics and transmitter release evoked by single action potentials at mammalian central synapses. Biophys J 72:637-651.

Thiagarajan TC, Lindskog M, Tsien RW (2005) Adaptation to synaptic inactivity in hippocampal neurons. Neuron 47:725-737.

Turrigiano GG (2008) The self-tuning neuron: synaptic scaling of excitatory synapses. Cell 135:422-435.

Turrigiano GG, Leslie KR, Desai NS, Rutherford LC, Nelson SB (1998) Activity-dependent scaling of quantal amplitude in neocortical neurons. Nature 391:892-896.

Wierenga CJ, Walsh MF, Turrigiano GG (2006) Temporal regulation of the expression locus of homeostatic plasticity. J Neurophysiol 96: $2127-2133$. 